\title{
Shell-model description of monopole shift in neutron-rich $\mathrm{Cu}$
}

\author{
N.A. Smirnova, A. De Maesschalck, A. Van Dyck, K. Heyde \\ Vakgroep Subatomaire en Stralingsfysica, Universiteit Gent, Proeftuinstraat 86, B-9000 Gent, Belgium
}

(Dated: November 22, 2018)

\begin{abstract}
Variations in the nuclear mean-field, in neutron-rich nuclei, are investigated within the framework of the nuclear shell model. The change is identified to originate mainly from the monopole part of the effective two-body proton-neutron interaction. Applications for the low-lying states in odd- $A$ $\mathrm{Cu}$ nuclei are presented. We compare the results using both schematic and realistic forces. We also compare the monopole shifts with the results obtained from large-scale shell-model calculations, using the same realistic interaction, in order to study two-body correlations beyond the proton mean-field variations.
\end{abstract}

PACS numbers: 21.10.-k, 21.30.-x,21.60.Cs

The nuclear shell model is based on the assumption that each of the nucleons in an atomic nucleus moves independently in an average field created by the other nucleons. One of the major successes of the model, even in its simplest version when the average potential is approximated by the the harmonic oscillator potential with the centrifugal term $(l \cdot l)$ and spin-orbit interaction $(l \cdot s)$, was the reproduction of the so-called magic numbers, i.e. the numbers of protons and neutrons which give additional stability to a nuclear system $(2,8,20,28,50,82,126)$. Inclusion of correlations beyond the mean-field approximation through the introduction of the residual two-body interaction provides us with an excellent description of atomic nuclei in the vicinity of the valley of $\beta$-stability 1, 22.

The shell structure of nuclei with large proton or neutron excess differs considerably from that of the stable nuclei. The important role in the mechanism of changes is played by the proton-neutron interaction as has long been recognized [3, 4]. It was demonstrated by the mean-field calculations [5] that the neutrons in very neutron-rich nuclei experience an average field with a much more diffused boundary due to the close-lying continuum and increased importance of the pairing interaction. As a result, the shell structure resembles that of a harmonic oscillator with the spin-orbit term only.

Within the shell-model concept, the description of the nuclear mean field is conventionally obtained by considering the so-called monopole Hamiltonian constructed from the centroids of the two-body interaction [6, 7, 8, [9]. The eigenvalues of this Hamiltonian were shown $[\underline{9}$ to provide the average energies of the specific spherical configurations. They are usually referred to as effective single-particle energies, and should reproduce the energies of single-proton (single-neutron) states in odd- $A$ nuclei with $Z(N)$ equal to a magic number plus or minus one proton (neutron).

Recent shell-model calculations of neutron-rich nuclei with masses below $A \approx 40$ studied the mechanisms of the changes in the shell-structure of the neutron mean-field [10, 11]. It was shown that an increasing $N / Z$ ratio, certain neutron orbitals move up or down thus deteriorating the usual shell closures and creating new ones. For example, the well-pronounced shell gap at $N=20$ in nuclei near ${ }^{40} \mathrm{Ca}$, disappears for nuclei with $Z<12$, while $N=16$ instead appears as a new magic number. This is a consequence of the crucial role played by the attractive proton-neutron effective nucleon-nucleon interaction (especially, in $T=0$ channel) in which spin-orbit partners are involved [4].

It can be expected that the proton-neutron interaction will influence in a major way the effective single-proton energies of the proton mean-field. It is well-known that the ordering and spacing of the proton mean-field orbitals in neutron-deficient or neutron-rich isotopes is not the same compared to $\beta$-stable nuclei, and may exhibit pronounced differences.

In this article, we intend to investigate the evolution of the proton mean-field from nuclei, situated around the valley of $\beta$-stability, towards very neutron-rich systems. In particular, we consider nuclei near semi-magic ones, namely, those which differ from a semi-magic system by an extra proton particle (hole). Typical examples are the F, Sc, $\mathrm{Cu}, \mathrm{Sb}$ and Bi isotopes.

The advantage of this choice is that the low-lying states in these nuclei usually carry a major part of the proton single-particle strength and thus a direct comparison between theory and experiment becomes possible. These nuclei can be rather well described by an inert core with one extra proton particle (hole) and a number of valence neutrons outside the core. We then estimate the effective single-particle energies $\left(\tilde{\epsilon}_{j}\right)$ in the proton mean-field as a bare singleparticle energy $\left(\epsilon_{j}\right)$ plus the average proton-neutron part of the interaction [12, 13, 14]. In this approximation, the evolution of the single-particle states as a function of neutron number will depend on the specific proton-neutron effective interactions $\left(\left\langle j_{\pi} j_{\nu}|V| j_{\pi} j_{\nu}\right\rangle\right)$ and the pairing properties amongst the neutrons.

The existence of the single-proton (single-neutron) shifts is well known experimentally in a series of isotopes (isotones) [15]. Recently a lot of new data has been obtained on odd- $A \mathrm{~F}$ [16, 17, 18], $\mathrm{Cu}$ 19] and $\mathrm{Bi}$ [20, 21] isotopes, up to very neutron-rich species.

In the present study we apply this approach to neutron-rich Cu-isotopes. We choose two types of the effective 
interaction, a schematic zero-range force and a realistic one, based on the G-matrix [22, 23], in order to test the sensitivity to the use of various (schematic versus realistic) forces. Finally, a comparison of the monopole shifts with the results of the large-scale shell-model diagonalization is carried out.

The interest in neutron-rich nuclei around ${ }^{68} \mathrm{Ni}$ and in particular the questioned magicity of ${ }^{78} \mathrm{Ni}$ has recently motivated the global study of low-energy nuclear structure for both odd- $A$ Cu nuclei [19] at the LISOL facility in Louvain-la-Neuve and odd-odd mass $\mathrm{Cu}$ at ISOLDE, Cern [24] up to very neutron-rich species. Figure 1 contains the selected states of odd- $A \mathrm{Cu}$ with spin and parity quantum numbers of the valence proton oscillator shell above $Z=28$ : $2 p_{3 / 2}, 1 f_{5 / 2}, 2 p_{1 / 2}, 1 g_{9 / 2}$. The ground state of odd- $A \mathrm{Cu}$-nuclei up to ${ }^{73} \mathrm{Cu}$ is $3 / 2^{-}$conform with the occupation of the $2 p_{3 / 2}$ oscillator orbital. As seen from Fig. 1 , the $5 / 2^{-}$state starts to drop drastically in energy for nuclei with $A>69$ and might be expected to become the ground state in heavy odd- $A \mathrm{Cu}$.

Since we are interested in the variation of the proton single-particle energies, it is consistent to compare our theoretical results with the experimental centroids of the $2 p_{3 / 2}, 1 f_{5 / 2}, 2 p_{1 / 2}$ and $1 g_{9 / 2}$ single-proton states, defined as

$$
E(n l j)=\frac{\sum_{k} S_{n l j}^{k} E_{n l j}^{k}}{\sum_{k} S_{n l j}^{k}}
$$

where $S_{n l j}^{k}$ are spectroscopic factors for a proton transfer (from $(n l j)$ single-particle state), and $E_{n l i}^{k}$ is the experimental excitation energy of the $k$ th state with $J=j$ (both taken from [25, 26, 27, 28, 29, 30] and Ref. [19]).

Unfortunately, the data on spectroscopic factors is rather scare and differs significantly from one experiment to another. In Table I we summarize the available spectroscopic strength for the states of interest in ${ }^{59-65} \mathrm{Cu}(\mathrm{see}$ captions of the tables for references). The experimental centroids are plotted in Fig. 2(a). For those states (isotopes) for which no experimental data exists, only the lowest states of a given spin is indicated. As seen from the table, the sum rule (for the transfer to both, $T_{f}=T_{i} \pm \frac{1}{2}$ states) is far from being accomplished, especially, for $9 / 2^{+}$states. Thus, one should be cautious with the trends of experimental "centroids" for this particular state.

The low-lying states of a nucleus with one proton (neutron) outside the doubly magic core can be considered as mainly single-particle states and they usually serve to obtain information on the single-particle energies $\epsilon_{j}$ used in the shell-model. Here $j$ stands for the quantum numbers $(n, l, j, m)$ of a given orbital.

The interaction between the valence proton and the filled neutron orbital results in a change of the single-particle energy that can be expressed as [4, 31]

$$
\tilde{\epsilon}_{j_{\pi}}=\epsilon_{j_{\pi}}+n_{\nu} E_{j_{\pi} j_{\nu}}
$$

where $n_{\nu}$ is the number of valence neutrons and $E_{j_{\pi} j_{\nu}}$ is the average matrix element of the proton-neutron interaction:

$$
E_{j_{\pi} j_{\nu}}=\frac{\sum_{J}\left\langle j_{\pi} j_{\nu} ; J|V| j_{\pi} j_{\nu} ; J\right\rangle(2 J+1)}{\sum_{J}(2 J+1)},
$$

with $V$ standing for the effective two-body interaction, and $J$ being the total angular momentum of a two-body configuration.

Taking into account that the model space for neutrons in general consists of several single-particle orbitals and that the pairing force spreads neutrons over these valence orbitals, one arrives at the following expression [12, 13, 14]:

$$
\tilde{\epsilon}_{j_{\pi}}=\epsilon_{j_{\pi}}+\sum_{j_{\nu}} E_{j_{\pi} j_{\nu}}\left(2 j_{\nu}+1\right) v_{j_{\nu}}^{2}
$$

where $v_{j_{\nu}}^{2}$ stands for the occupation probability of the orbital $j_{\nu}$ and the summation is performed over all valence neutron orbitals.

From eq. (4) it is obvious that the proton-neutron interaction causes the proton single-particle states to change, depending on its character (attraction or repulsion) and magnitude. The larger the overlap between the proton and neutron radial wave functions and the higher $j_{\nu}$ of a given neutron orbital is, the stronger the variations are.

Remark that only the monopole component from the multipole representation of the effective force contributes to the average matrix element (3) [14]. This is why the effect is referred to as the monopole shift.

The basic ingredients required in (4) are: (i) the shell-model space; (ii) the effective proton-neutron interaction, and (iii) neutron occupation probabilities. 
For the description of the $\mathrm{Cu}$-nuclei we have chosen the shell-model space consisting of four valence orbitals, $2 p_{3 / 2}$, $1 f_{5 / 2}, 2 p_{1 / 2}$, and $1 g_{9 / 2}$, both for neutrons and protons, outside ${ }^{56} \mathrm{Ni}$-core. This model space contains all necessary degrees of freedom to describe low energy nuclear structure.

In the present work we have restricted ourselves to two types of the effective interaction. First, we consider the zero-range $\delta$-interaction with the spin-exchange term [32]

$$
V=-V_{0} \delta\left(\vec{r}_{\pi}-\vec{r}_{\nu}\right)\left(1-\alpha+\alpha \vec{\sigma}_{\pi} \vec{\sigma}_{\nu}\right)
$$

The two parameters, the overall strength $V_{0}$ and the strength of the exchange term $\alpha$ are to be adjusted to the experimental data in odd-odd nuclei from the mass region one is studying.

The geometrical properties of the zero-range interaction, even including spin-exchange contributions (5), allow to obtain a very simple analytical expression for the monopole shift:

$$
\tilde{\epsilon}_{j_{\pi}}=\epsilon_{j_{\pi}}-V_{0}(1-\alpha) \sum_{j_{\nu}} F_{n_{\pi} l_{\pi} ; n_{\nu} l_{\nu}}^{0}\left(2 j_{\nu}+1\right) v_{j_{\nu}}^{2} .
$$

where $F_{n_{\pi} l_{\pi} ; n_{\nu} l_{\nu}}^{0}$ are Slater integrals. In order to derive this result, we had to make use of a peculiar property of the Wigner $3 j$-symbols, as described in Appendix A.

We stress the fact that since the average proton-neutron matrix element from the $\delta$-interaction entering (6) does not depend on $j_{\pi}$, the relative shift for spin-orbit partners (see e.g. the result for $2 p_{1 / 2}$ and $2 p_{3 / 2}$ orbitals in Fig. 2 (b)) stays constant and remains equal to the difference of the original single-particle energies $\left(\epsilon_{j_{\pi}}-\epsilon_{j_{\pi}^{\prime}}\right)$.

For the $\mathrm{Cu}$-isotopes, we have determined the parameters of (5) to be $V_{0}=400 \mathrm{MeV}$.fm ${ }^{3}$ and $\alpha=0.1$. These values suit the systematics known from the description of heavier isotopes [14] and they result in two-body matrix elements of the same order as those of the realistic interaction which will be described below. The chosen interaction also gives a reasonable, although slightly stretched, spectrum of ${ }^{58} \mathrm{Cu}[33]$.

The second type of interaction used here is a realistic one, obtained from the $G$-matrix through a Brueckner-HartreeFock procedure [22] and modified further for a monopole correction [23] following the recipe of Ref. [7] to account for the saturation properties. The interaction works very well in the description of Ni and Cu-isotopes, reproducing many known spectroscopic properties [23].

The neutron occupation probabilities $v_{j_{\nu}}^{2}$ have been obtained from the solution of the BCS equations using a pairing Hamiltonian [32]:

$$
H=-G \sum_{j m, j^{\prime} m^{\prime}} a_{j m}^{\dagger} a_{j,-m}^{\dagger} \tilde{a}_{j^{\prime} m^{\prime}} \tilde{a}_{j^{\prime},-m^{\prime}} .
$$

The strength of the pairing interaction was chosen to reproduce the pairing gaps in neighboring even-even Niisotopes [34] resulting in a dependence $G=23 / A \mathrm{MeV}$.

In Fig. 2 we compare the experimental energy centroids (Fig. 2(a)) with the results of the calculations according to eq. (4) with two types of the interaction (Fig. 2(b,c)).

Figure 2(b) contains the results obtained using the $\delta$-force. For ${ }^{57} \mathrm{Cu}$, the lowest states are given by the empirical values $\epsilon_{j_{\pi}}$. These values are modified by the two-body interaction. Effective single-proton energies for the $1 f_{5 / 2}$ and $1 g_{9 / 2}$ states first move up, when neutrons are filling the $p f$ orbitals, and then (from ${ }^{69} \mathrm{Cu}$ ) go down relative to the $2 p_{3 / 2}$ state as the neutron $1 g_{9 / 2}$ orbital starts to be filled. This is governed by the relative magnitude of the centroids of the interaction. In particular, the drastic lowering of the $1 f_{5 / 2}$ and $1 g_{9 / 2}$ orbitals stems from more attractive $E_{1 f_{5 / 2} 1 g_{9 / 2}}$ and $E_{1 g_{9 / 2} 1 g_{9 / 2}}$ values relative to $E_{2 p_{3 / 2} 1 g_{9 / 2}}$. The energy of the $2 p_{1 / 2}$ orbital stays unchanged with respect to the $2 p_{3 / 2}$ ground state as has been mentioned before. At $A=79$ we observe a crossing between the effective $2 p_{1 / 2}$ and $1 f_{5 / 2}$ states, however, the chosen interaction does not reproduce the expected crossing of the effective $2 p_{3 / 2}$ and $1 f_{5 / 2}$ orbitals.

In Fig. 2(c), we present the results obtained with a realistic effective interaction described above. Only diagonal proton-neutron matrix elements contribute to the expression (4). Though the overall trend obtained from both types of the interaction remains the same, as follows from the similar character of the matrix elements, there are certain distinctions. First, the energy of the $2 p_{1 / 2}$ orbital varies relative to the $2 p_{3 / 2}$ ground state orbital (see Fig. 2 (c)) . Secondly, the proton $1 f_{5 / 2}$ orbital decreases steeper as neutrons are added in the $1 g_{9 / 2}$ orbital and for ${ }^{79} \mathrm{Cu}$ the inversion of the $1 f_{5 / 2}$ and $2 p_{3 / 2}$ orbitals is predicted, compared to the use of the $\delta$-interaction.

We point out that the lack of agreement between the variation of the effective $1 g_{9 / 2}$ single-proton state and the experimental $9 / 2^{+}$level is due to the fact that the single-particle strength of the $1 g_{9 / 2}$ state lies higher up and the 
lowest state which is plotted in Fig. 2(a), due to the lack of experimental data, contains only a small fraction of that (see Table I).

In order to get an insight into additional correlations that cause a variation of the single-particle centroids of $2 p_{3 / 2}$, $1 f_{5 / 2}, 2 p_{1 / 2}$ and $1 g_{9 / 2}$ proton states beyond the monopole shift, we have carried out large-scale shell-model calculations using the same realistic effective interaction as described above. The model space consists of the $\left(1 f_{5 / 2} 2 p_{3 / 2} 2 p_{1 / 2} 1 g_{9 / 2}\right)$ orbitals outside the ${ }^{56} \mathrm{Ni}$-core, truncated in case of ${ }^{67} \mathrm{Cu},{ }^{65,69} \mathrm{Cu}$ and ${ }^{71} \mathrm{Cu}$ (up to 3,4 and 8 particles, respectively, were allowed to occupy the $1 g_{9 / 2}$ orbital). The calculations were done using the shell-model code Oxbash [35]. The single-particle centroids for the $2 p_{3 / 2}, 1 f_{5 / 2}, 2 p_{1 / 2}$ and $1 g_{9 / 2}$ states obtained by taking into account up to about 400800 excited states weighted by their spectroscopic factors (in order to exhaust the sum rule for $T_{f}=T_{i}-\frac{1}{2}$ transfer up to $98 \%$ or to get a reasonable saturation of the total value for truncated spaces) are shown in Fig. 2(d). The wave functions of the corresponding $\mathrm{Ni}$-isotopes have been calculated without any restrictions of the model space.

A remarkable result is that the centroids shown in Fig. 2(d) are rather close to those obtained by the analytical

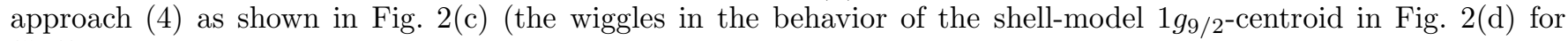
${ }^{65-69} \mathrm{Cu}$ are related most probably to the truncations of the model space which is otherwise too large to be treated numerically). The monopole energy shifts incorporates the effects of a certain class of residual interactions (self-energy correction of the proton single-particle energy because of proton-neutron interactions and neutron pairing) on the bare proton single-particle energy within the model space, as expressed by eq. (4).

A comparison of these results thus illuminates the question what type of correlations is important in the calculation of the energy shifts beyond the monopole shift. A rather good agreement between the centroids of Figs. 2(c) and 2(d) confirms that the basic ingredients of the single-particle centroid variations are already taken into account by eq. (4). This is a most interesting and valuable result.

To illustrate the importance of the pairing effects, we plot in Fig. 2(e) the effective single-proton energies obtained from the monopole Hamiltonian with normal filling of the single-particle orbitals by neutrons (the approach chosen in Refs. 6, 7, 9, 10, 11]). It is seen that through the occupation probabilities, the pairing force (Fig. 2(c)) smoothes significantly the single-particle behavior. Fig. 2(f) shows the same as Fig. 2(e), but with a different order of filling of neutrons orbitals (neutron $2 p_{1 / 2}$ is filled before neutron $1 f_{5 / 2}$ ). The behavior of single-particle orbitals does not have a zigzag structure in this case, but the maximum of the centroids is shifted to ${ }^{63} \mathrm{Cu}$, compared to the calculations with pairing effects (Fig. 2(b,c)). Let us note that physically the order of filling chosen in Fig. 2(e) is more realistic than that implied in Fig. 2(f), as follows from the experimental spectra of ${ }^{67,69} \mathrm{Ni}$ (neutron single-particle orbital $2 p_{1 / 2}$ is higher than $1 f_{5 / 2}$ in these nuclei).

Since the lowest-lying $J^{\pi}=1 / 2^{-}, 3 / 2^{-}, 5 / 2^{-}$and $9 / 2^{+}$states represent only a fraction of the total proton singleparticle strength, we compare the energies of the lowest $1 / 2^{-}, 3 / 2^{-}, 5 / 2^{-}$and $9 / 2^{+}$experimental states (Fig. 3(a)) and theoretical states obtained from the large-scale diagonalization (Fig. 3(b)). In general, we remark a very good agreement with the experimental data due to the quality of the effective interaction.

By comparing the results of Figs. 2(c,d) and 3(b), it is clear that the correlations beyond the monopole approximation are necessary to understand a detailed fine structure of the energy variations for the lowest $1 / 2^{-}, 5 / 2^{-}$and $9 / 2^{+}$ levels. This is particularly so for the $1 / 2^{-}$and $5 / 2^{-}$levels where specific kinks, related to a possible shell closure at $N=40$, are clearly seen in the diagonalization. The general trend of the $9 / 2^{+}$level, as obtained from diagonalization, is quite different compared to the monopole shift. These effects are obviously a result of interactions of the pure single-particle configurations with many of the excited configurations with the same $J^{\pi}$ value.

Finally, in Fig. 4 we summarize the spectroscopic factors of the $2 p_{3 / 2}, 1 f_{5 / 2}, 2 p_{1 / 2}$ and $1 g_{9 / 2}$ proton stripping from the ${ }^{A-1} \mathrm{Ni}$-core into the lowest-lying $1 / 2^{-}, 3 / 2^{-}, 5 / 2^{-}$and $9 / 2^{+}$states of ${ }^{A} \mathrm{Cu}$, respectively, as provided by the shell-model diagonalization. The spectroscopic factors have been normalized to the sum rule presented in Appendix B. The absolute spectroscopic factors for the lowest states of a given spin are shown in Table I in comparison with the available experimental data on ${ }^{59-65} \mathrm{Cu}$. The calculated spectroscopic factors are in rather good agreement for the lowest $3 / 2^{-}$and $1 / 2^{-}$states (within $20 \%$ ), however, the disagreement increases for $5 / 2^{-}$and $9 / 2^{+}$states in these isotopes. It is not clear, at present, what the origin of these differences is. It may be due to a lack of experimental data, but it may also point out certain deficiencies in the realistic interaction presently used. As for the whole series of $\mathrm{Cu}$-isotopes, the calculations show that only the $3 / 2^{-}$ground state remains more or less a pure single-particle state. The lowest $1 / 2^{-}$state is predicted to carry about half of the $2 p_{1 / 2}$ single-particle strength. The lowest $5 / 2^{-}$state shows much more mixing for ${ }^{61-69} \mathrm{Cu}$. This is in line with experimental observations of a few $5 / 2^{-}$states in these isotopes even at low energies. Finally, the $9 / 2^{+}$state is predicted to be a rather pure single-particle $1 g_{9 / 2}$ state only for light isotopes. Starting from ${ }^{67} \mathrm{Cu}$, the single-proton strength almost vanishes. In order to test these predictions, more precise data on spectroscopic factors and level spin and parity assignments would be very helpful.

In conclusion, it becomes clear that using both simple effective and realistic forces in order to derive the corresponding monopole energy shifts, the experimental proton single-particle centroids are, at present, only moderately described. This comparison is hampered by the lack of reliable and extensive experimental results on one-nucleon 
transfer reactions.

An interesting point is a reproduction of a lowering in the $1 f_{5 / 2}$ energy centroid, once the $N=40$ neutron number is passed, in particular using realistic forces. We also remark that correlations that define the monopole energy shift, as defined in eq. (4), are very much consistent with the full theoretical single-particle energy centroid resulting from a diagonalization of the full energy matrix, encompassing all possible residual interactions within the large model space. This result is an interesting one and has to be studied in other mass regions, too. Although the energies obtained from the diagonalization in the large model space concerning the lowest state of each spin, parity, following rather well the corresponding experimental energies and exhibit interesting fine structure, the theoretical centroid, using a significant number of the eigenstates, becomes rather smooth again. At the same time, more experimental data on structure of nuclei from the region would serve a good testing ground for the present results and the interactions used.

\section{Acknowledgments}

N.A.S. thanks E. Caurier and F. Nowacki from IReS (Strasbourg) for making available the interaction exploited here, and for useful discussions. We thank R.F. Casten for interesting discussions. This work was supported by the Inter-University Attraction Poles (IUAP) under project P5/07. K.H. is grateful to the FWO-Vlaanderen for financial support.

\section{Appendix A: Properties of the $\delta$-interaction}

The diagonal matrix element of the interaction (5) between the two-body states in $j j$-coupling reads

$$
\left\langle j_{\pi} j_{\nu} ; J|V| j_{\pi} j_{\nu} ; J\right\rangle=-V_{0}\left(2 j_{\pi}+1\right)\left(2 j_{\nu}+1\right)\left(\left(1-2 \alpha-2 \alpha(-1)^{J}\right)\left(\begin{array}{ccc}
j_{\pi} & j_{\nu} & J \\
1 / 2 & -1 / 2 & 0
\end{array}\right)^{2}+\left(\begin{array}{ccc}
j_{\pi} & j_{\nu} & J \\
1 / 2 & 1 / 2 & -1
\end{array}\right)^{2}\right) .
$$

In order to evaluate the average matrix element,

$$
E_{j_{\pi} j_{\nu}}=\frac{\sum_{J}\left\langle j_{\pi} j_{\nu} ; J|V| j_{\pi} j_{\nu} ; J\right\rangle(2 J+1)}{\sum_{J}(2 J+1)},
$$

using the above two-body proton-neutron matrix elements, we have to exploit the well-known summation properties for $3 j$-symbols,

$$
\begin{aligned}
& \sum_{J}(2 J+1)\left(\begin{array}{ccc}
j_{\pi} & j_{\nu} & J \\
1 / 2 & -1 / 2 & 0
\end{array}\right)^{2}=1 \\
& \sum_{J}(2 J+1)\left(\begin{array}{ccc}
j_{\pi} & j_{\nu} & J \\
1 / 2 & 1 / 2 & -1
\end{array}\right)^{2}=1
\end{aligned}
$$

Besides, in the calculations the particular expression,

$$
\sum_{J}(-1)^{J}(2 J+1)\left(\begin{array}{ccc}
j_{\pi} & j_{\nu} & J \\
1 / 2 & -1 / 2 & 0
\end{array}\right)^{2}
$$

appears for which we have found no standard result in the literature on angular momentum algebra (e.g., Ref. [36]). We had to be able to establish that the above expression (12) vanishes, as in the most general case,

$$
\sum_{J}(-1)^{J}(2 J+1)\left(\begin{array}{ccc}
j & j^{\prime} & J \\
m & -m & 0
\end{array}\right)^{2}=0,
$$

due to the peculiar summation properties of the considered $3 j$-symbols which we would like to mention here:

$$
\sum_{J=e v e n}(-1)^{J}(2 J+1)\left(\begin{array}{ccc}
j & j^{\prime} & J \\
m & -m & 0
\end{array}\right)^{2}=1 / 2,
$$




$$
\sum_{J=o d d}(-1)^{J}(2 J+1)\left(\begin{array}{ccc}
j & j^{\prime} & J \\
m & -m & 0
\end{array}\right)^{2}=-1 / 2
$$

Such summations on the odd or even values of the angular momentum are often encountered in quantum mechanics.

As a result, we obtain for the average matrix element of the proton-neutron interaction the following analytical expression:

$$
E_{j_{\pi} j_{\nu}}=-V_{0} F_{n_{\pi} l_{\pi} ; n_{\nu} l_{\nu}}^{0}(1-\alpha)
$$

where $F_{n_{\pi} l_{\pi} ; n_{\nu} l_{\nu}}^{0}$ stands for the Slater integral.

Remark, that the average matrix element does not depend on the $j$ quantum number of the orbitals involved, as well as it vanishes for the spin-exchange term.

In a similar way, we can evaluate the average particle-hole matrix element. The particle-hole matrix element relates to the particle-particle one via the Pandya transformation [32]:

$$
\left\langle j_{\pi} j_{\nu}^{-1} ; J|V| j_{\pi} j_{\nu}^{-1} ; J\right\rangle=-\sum_{J^{\prime}}\left(2 J^{\prime}+1\right)\left\{\begin{array}{ccc}
j_{\pi} & j_{\nu} & J^{\prime} \\
j_{\pi} & j_{\nu} & J
\end{array}\right\}\left\langle j_{\pi} j_{\nu} ; J|V| j_{\pi} j_{\nu} ; J\right\rangle
$$

Exploiting the following summation properties for $3 j$-symbols,

$$
\begin{aligned}
& \sum_{J^{\prime}}\left(2 J^{\prime}+1\right)\left\{\begin{array}{lll}
j_{\pi} & j_{\nu} & J^{\prime} \\
j_{\pi} & j_{\nu} & J
\end{array}\right\}\left(\begin{array}{ccc}
j_{\pi} & j_{\nu} & J \\
1 / 2 & -1 / 2 & 0
\end{array}\right)^{2}=\left(\begin{array}{ccc}
j_{\pi} & j_{\nu} & J \\
1 / 2 & 1 / 2 & -1
\end{array}\right)^{2}, \\
& \sum_{J^{\prime}}\left(2 J^{\prime}+1\right)\left\{\begin{array}{lll}
j_{\pi} & j_{\nu} & J^{\prime} \\
j_{\pi} & j_{\nu} & J
\end{array}\right\}\left(\begin{array}{ccc}
j_{\pi} & j_{\nu} & J \\
1 / 2 & 1 / 2 & -1
\end{array}\right)^{2}=\left(\begin{array}{ccc}
j_{\pi} & j_{\nu} & J \\
1 / 2 & -1 / 2 & 0
\end{array}\right)^{2},
\end{aligned}
$$

and making use of the peculiar relation (12), one obtains for the average particle-hole matrix element the result

$$
E_{j_{\pi} j_{\nu}^{-1}}=V_{0} F_{n_{\pi} l_{\pi} ; n_{\nu} l_{\nu}}^{0}(1-\alpha) .
$$

This expression differs from that for a particle-particle average matrix element only by its sign.

\section{Appendix B: Sum rule for the spectroscopic factors}

The total spectroscopic strength in proton stripping reactions to the states with $T_{f}=T_{i}-\frac{1}{2}$ is given by the following sum rule [37]:

$$
C^{2} \sum_{f} \frac{2 J_{f}+1}{2 J_{i}+1} S_{f}(n l j)=\frac{T_{i}+T_{i z}}{2 T_{i z}\left(2 T_{i}+1\right)}\left[\left(T_{i}+T_{i z}+1\right)\left\langle\tilde{n}_{\pi}(j)\right\rangle_{J_{i}, T_{i}}-\left(T_{i}-T_{i z}+1\right)\left\langle\tilde{n}_{\nu}(j)\right\rangle_{J_{i}, T_{i}}\right]
$$

$J_{i}, J_{f}$ are the spin and $T_{i}, T_{f}$ are the isospin quantum numbers of the initial and final states (in ${ }^{A-1} \mathrm{Ni}$ and ${ }^{A} \mathrm{Cu}$, respectively), $C=\left(T_{i}, T_{i z}, \frac{1}{2},-\frac{1}{2} \mid T_{f}=T_{i}-\frac{1}{2}, T_{f z}=T_{i z}-\frac{1}{2}\right)$ is the Clebsch-Gordan coefficient, $\langle\tilde{n}(j)\rangle$ is the average number of proton or neutron holes in the $j$-orbital in the initial state (ground state of ${ }^{A-1} \mathrm{Ni}$ in our case).

Since in the case considered here, $J_{i}=0, J_{f}=j$, the sum rule (21) reduces to

$$
C^{2} \sum_{f} S_{f}(n l j)\left(2 J_{f}+1\right)=\left\langle\tilde{n}_{\pi}(j)\right\rangle_{T_{i}}-\frac{1}{2 T_{i}+1}\left\langle\tilde{n}_{\nu}(j)\right\rangle_{T_{i}}
$$

as indicated in Table I where the average number of neutron holes in the ground state of Ni-isotopes are taken from the shell-model diagonalization.

[1] B. A. Brown and B. H. Wildenthal, Ann. Rev. Nucl. Part. Sci. 38, 29 (1988).

[2] E. Caurier, F. Nowacki, A. P. Zuker et al, Nucl. Phys. A 654, 747 (1999).

[3] A. de-Shalit and M. Goldhaber, Phys. Rev. 92, 1211 (1953).

[4] P. Federman and S. Pittel, Phys. Rev. C 20, 820 (1979). 
[5] J. Dobaczewski, I. Hamamoto, W. Nazarewicz, J. A. Sheikh, Phys. Rev. Lett. 72, 981 (1994).

[6] R.K.Bansal and J.B.French, Phys. Lett. 11, 145 (1964).

[7] A. Poves and A. P. Zuker, Phys. Rep. 70, 235 (1981).

[8] K. Heyde, C. De Coster, J. Schietse, Phys. Rev. C 49, 2499 (1994).

[9] M. Dufour and A.P.Zuker, Phys. Rev. C 54, 1641 (1996).

[10] T. Otsuka, R. Fujimoto, Y. Utsuno, B. A. Brown, M. Honma, T. Mizusaki, Phys. Rev. Lett. 87, 082502 (2001).

[11] T. Otsuka, Y. Utsuno, R. Fujimoto, B. A. Brown, M. Honma, T. Mizusaki, Eur. Phys. J. A 13, 69 (2002).

[12] A. L. Goodman, Nucl. Phys. A 287, 1 (1977).

[13] R. A. Sorensen, Nucl. Phys. A 420, 221 (1984).

[14] K. Heyde et al, Nucl. Phys. A 466, 189 (1987).

[15] S. Franchoo, Ph. D. thesis, University of Leuven (1999).

[16] H. Sakurai et al, Phys. Lett. B 448, 180 (1999).

[17] M. Thoennessen et al, Phys. Rev. C 68, 044318 (2003).

[18] W.A. Peters et al, Phys. Rev. C 68, 034607 (2003).

[19] S. Franchoo et al, Phys. Rev. C 64, 054308 (2001).

[20] J. Kurpeta et al, Eur. Phys. J. A 18, 31 (2003).

[21] H. De Witte et al, submitted.

[22] M. Hjorth-Jensen, T.T.S. Kuo, E. Osnes, Phys. Rep. 261, 125 (1995).

[23] F. Nowacki, Ph.D. thesis, IReS, Strasbourg, 1996; F. Nowacki, private communication.

[24] J. Van Roosbroeck et al, Phys. Rev. C (in press); J. Van Roosbroeck et al, Phys. Rev. Lett. (in press).

[25] http://www.nndc.bnl.gov

[26] C.M. Baglin, Nucl. Data Sheets 95, 215 (2002).

[27] M.R. Bhat, Nucl. Data Sheets 88, 417 (1999).

[28] B. Erjun, H. Junde, Nucl. Data Sheets 92, 147 (2001).

[29] N.J. Ward, J.K. Tuli, Nucl. Data Sheets 47, 135 (1986).

[30] B Zeidman, J.A. Nolen, Jr, Phys. Rev. C 18, 2122 (1978).

[31] A. de-Shalit and I. Talmi, Nuclear shell theory, (Academic Press, New York, 1963).

[32] K. Heyde, The Nuclear Shell Model, Study Edition, (Springer-Verlag, Berlin, Heidelberg, New-York, 1994)

[33] A.F. Lisetskiy, N. Pietralla, M. Honma et al, Phys. Rev. C 68, 034316 (2003).

[34] A.M. Oros-Puesquens and P.F. Mantica, Nucl. Phys. A 669, 8 (2000).

[35] B. A. Brown et al, The computer code Oxbash, MSU-NSCL Report No. 524 (1984).

[36] D.A. Varshalovich, A.N. Moskalev, V.K. Khersonskii, Quantum Theory of Angular Momentum, (World Scientific, 1988).

[37] P. J. Brussaard, P. W. M. Glaudemans, Shell-Model Applications in Nuclear Spectroscopy (Amsterdam: North-Holland Publishing, 1977). 
TABLE I: Available experimental data on spectroscopic factors of the lowest $3 / 2^{-}, 5 / 2^{-}, 1 / 2^{-}$and $9 / 2^{+}$states in ${ }^{59,61,63,65} \mathrm{Cu}$ (taken from taken from $\mathrm{Ni}(\mathrm{d}, \mathrm{n})$ or $\mathrm{Ni}\left({ }^{3} \mathrm{He}, \mathrm{d}\right)$ proton-stripping reaction studies [25, 26, 27, 28, 29, 30]). The sum rule refers to the proton transfer to both, $T_{f}=T_{i} \pm \frac{1}{2}$ states, while the shell-model sum rule is given for the states with $T_{f}=T_{i}-\frac{1}{2}$ only. The calculated spectroscopic factors for the lowest states of a given spin are indicated as well.

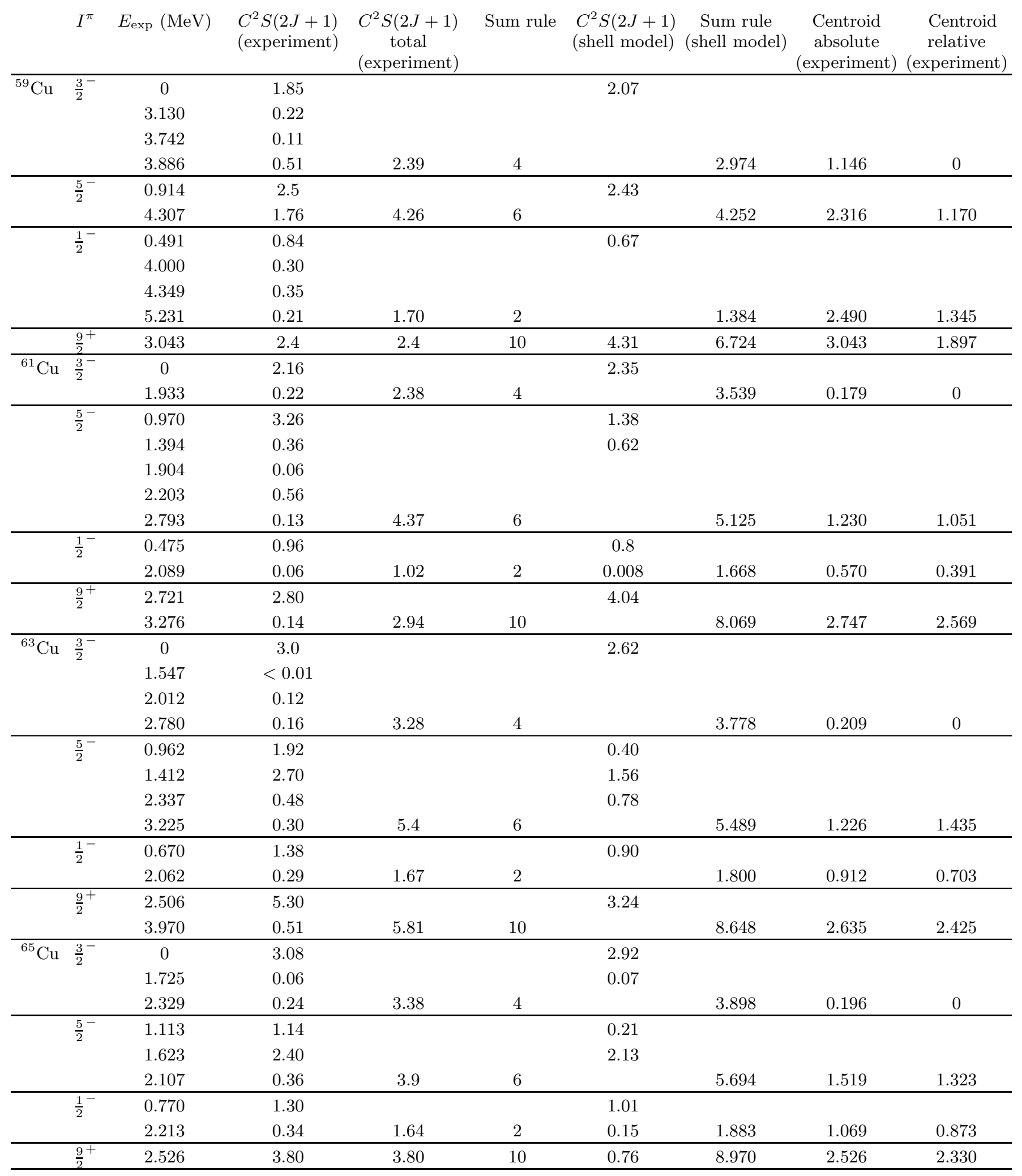




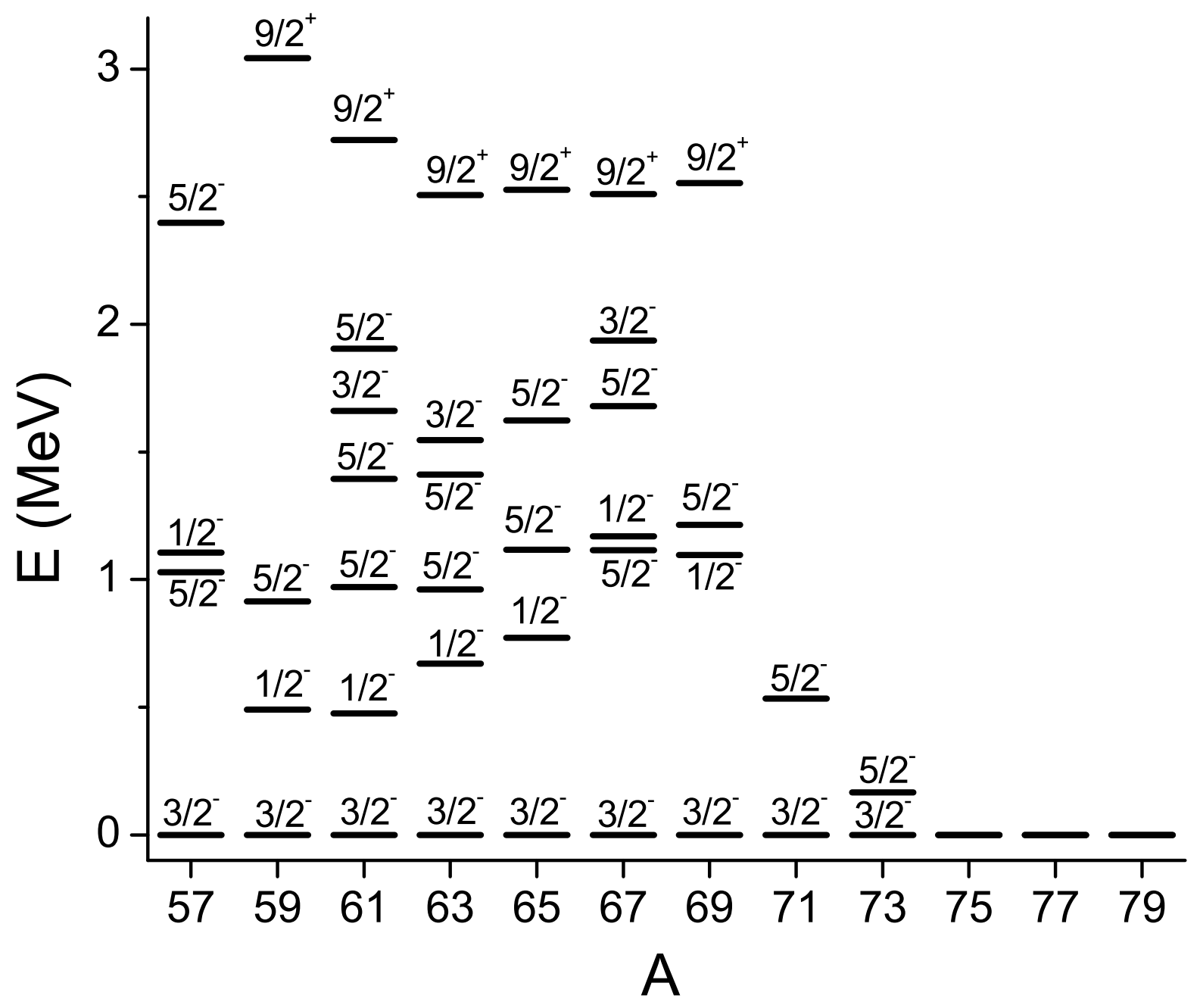

FIG. 1: Experimental low-lying states in odd- $A \mathrm{Cu}$ isotopes [19, 25]. 

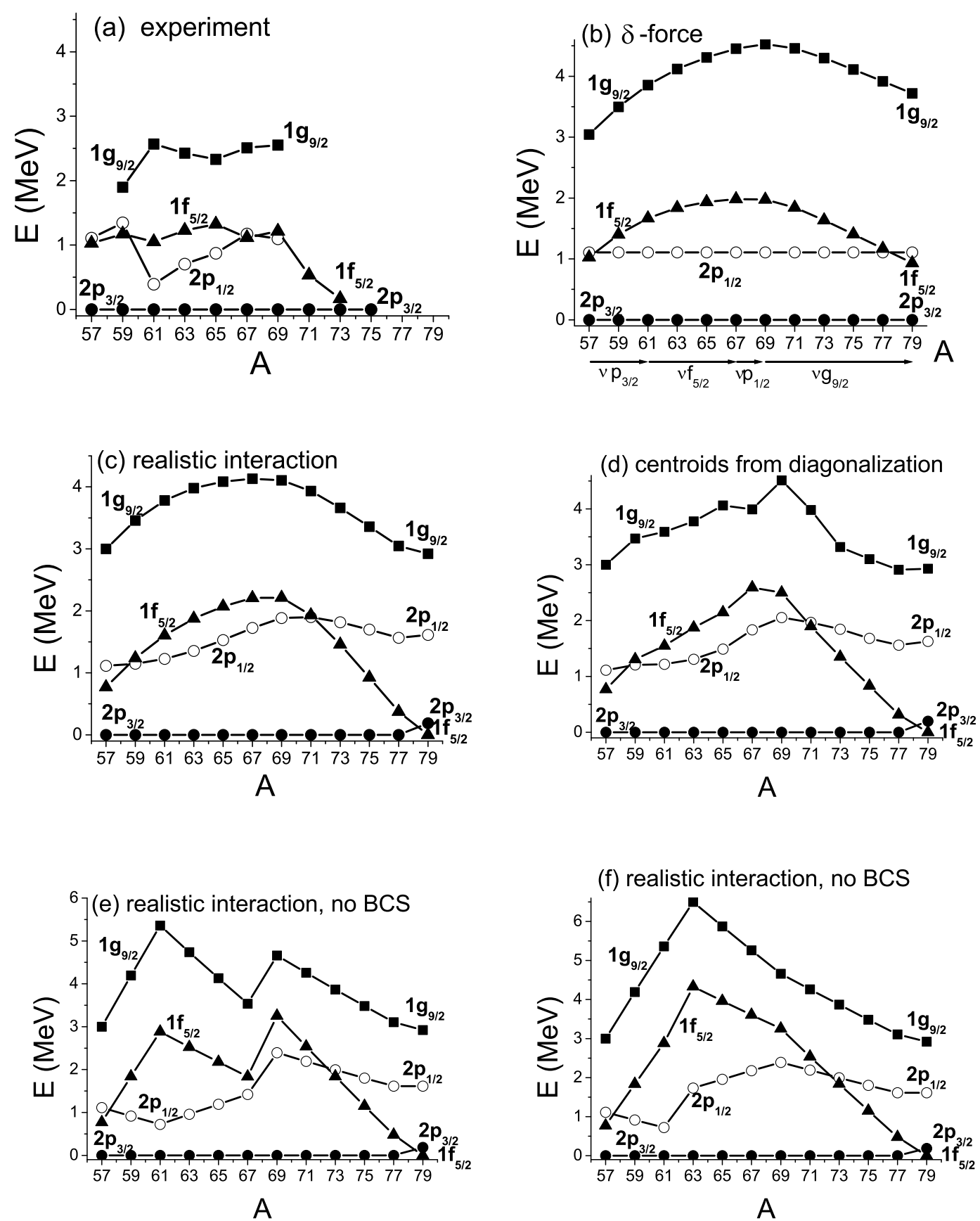

(f) realistic interaction, no BCS

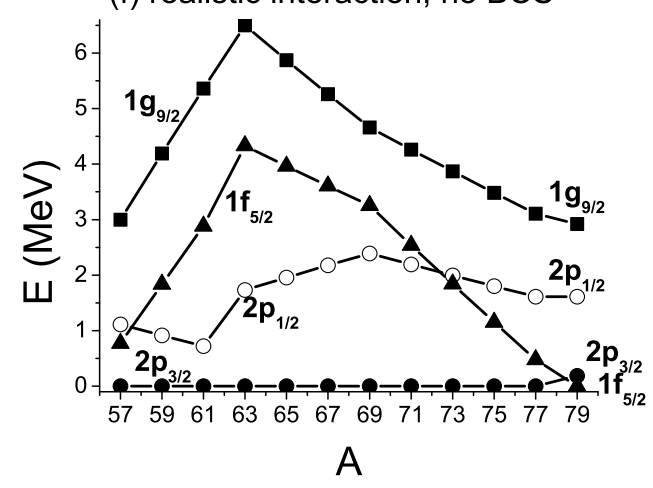

FIG. 2: (a) Experimental centroids when data is available (see Table I) or lowest states of a given spin in odd- $A$ Cu isotopes; (b,c) theoretical evolution of the single-particle states in odd- $A \mathrm{Cu}$ isotopes due to the monopole part of the proton-neutron $\delta$-interaction and realistic interaction, respectively; (d) shell-model centroids derived from the exact diagonalization, using a realistic interaction; (e,f) the same as in (c), but implying normal filling of the neutron orbitals (two possible ways of filling). The arrows in figure (b) below the plot indicate schematically the normal filling of neutron single-particle orbitals in Cu-isotopes. See text for details. 

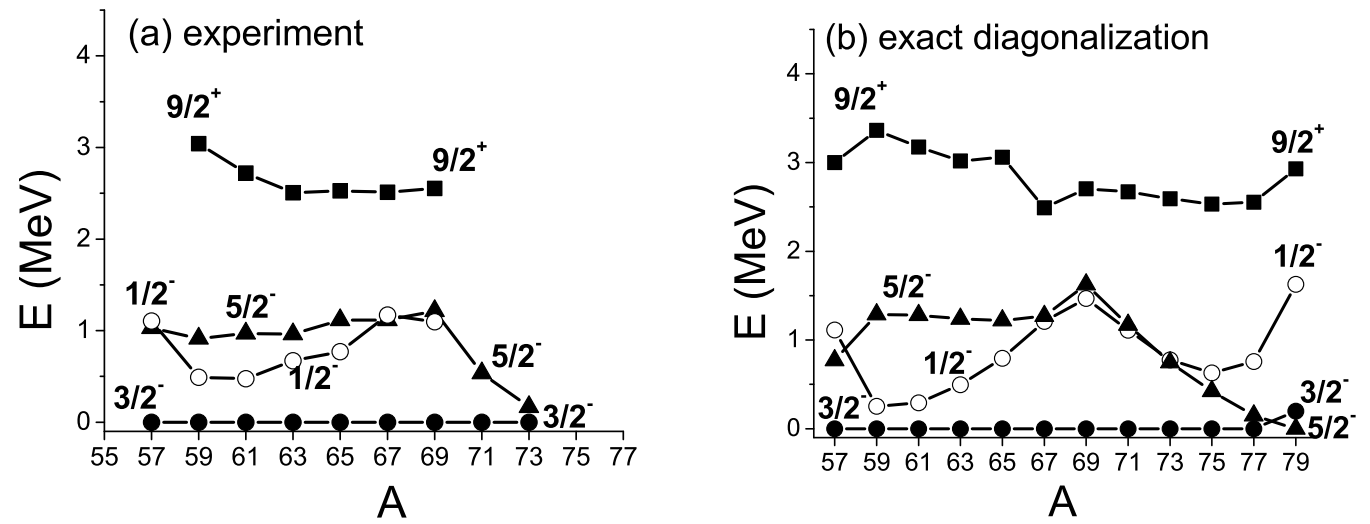

FIG. 3: The lowest $3 / 2^{-}, 5 / 2^{-}, 1 / 2^{-}$and $9 / 2^{+}$states in odd- $A$ Cu isotopes: (a) experimental values; (b) as obtained from the shell-model diagonalization with the realistic interaction. See text for details.

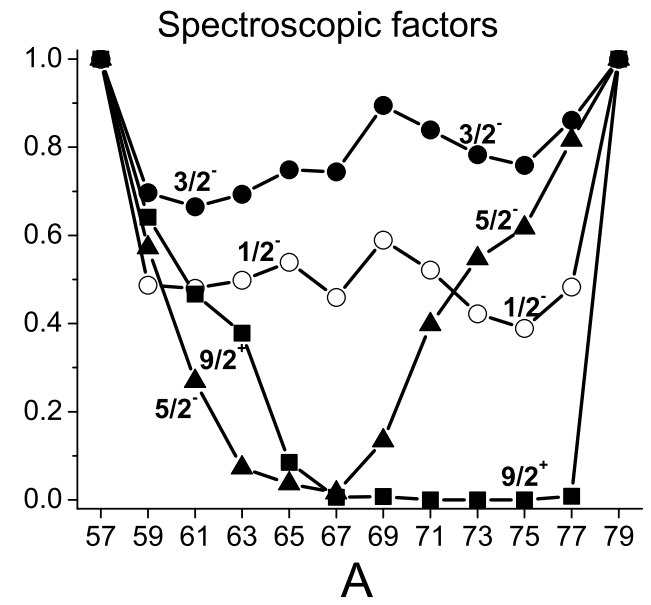

FIG. 4: Proton stripping spectroscopic factors into the lowest $3 / 2^{-}, 5 / 2^{-}, 1 / 2^{-}$and $9 / 2^{+}$states, respectively, in odd- $A$ $\mathrm{Cu}$ isotopes obtained from the shell-model diagonalization with the realistic interaction. The values are normalized to the shell-model sum rule. See text for details. 